\title{
CONSISTENCIA EN EL DISEÑO: PREDICCIÓN DE LA VELOCIDAD DE OPERACIÓN EN CARRETERAS
}

\author{
John Jairo Posada-Henaoํㅜ, Sara Cadavid-Agudelo², Laura Castro Gómez ${ }^{3}$ \\ ${ }^{1}$ Profesor asociado en la Facultad de Minas, departamento de Ingeniería Civil \\ Correo electrónico: jjposada@unal.edu.co \\ 2 Ingeniera civil, estudiante de la Maestría en Ingeniería, Infraestructura y Sistemas de Transporte \\ ${ }^{3}$ Ingeniera civil, estudiante de la Especialización en Vías y Transporte \\ Universidad Nacional de Colombia, sede Medellín
}

Recibido: 20 de febrero del 2014. Aprobado: 25 de julio del 2014.

Cómo citar este artículo: J.J. Posada-Henao, S. Cadavid-Agudelo y L. Castro-Gómez, "Consistencia en el diseño. Predicción de la velocidad de operación en carreteras". Ingeniería Solidaria, vol. 10, n. ${ }^{\circ}$ 17, pp. 39-47, en.-dic., 2014. doi: http://dx.doi.org/10.16925/in.v9i17.803

Resumen. La consistencia es un concepto utilizado, entre otros, para evaluar parte de la seguridad en las carreteras, tanto en la fase de diseño como en la de operación; por ejemplo, identificando la variación de la velocidad de operación con respecto a la velocidad de diseño a lo largo de una carretera. En este artículo se presenta el concepto de consistencia en el diseño de carreteras y sus diferentes enfoques, mostrando los principales modelos que se han aplicado y que se aplican actualmente según el perfil de velocidad, el cual se basa en la velocidad de operación a la cual se observa que los conductores operan sus vehículos en condiciones de flujo libre a lo largo de la carretera. Con el fin de simplificar los modelos de predicción de velocidad de operación, pero sin perder la calidad de los resultados, se presenta una propuesta para unificar varios de los modelos existentes que posean características similares, obteniendo como resultado altos coeficientes de correlación que soportan su validez. Además, se hacen unas recomendaciones para desarrollar futuros modelos aplicados a las condiciones colombianas para carreteras con calzada sencilla bidireccional o unidireccional y para dobles calzadas.

Palabras clave: consistencia, diseño geométrico, velocidad de operación, velocidad de diseño, perfil de velocidad.

\section{Consistency in Design: Prediction of THE Operative SPEed on RoAds}

\begin{abstract}
Consistency is a concept that is used, among others, to assess road safety, in both the design and operational phases; for example, identifying changes in the operative speed with respect to speed of design along the length of a road. This article presents the concept of consistency in road design and its different approaches, showing the model principles that have been applied and are currently applied according to the speed profile. This is based on the operative speed observed when people drive their vehicles under free-flowing conditions along a road. In order to simplify operative speed prediction models, but without losing sight of the quality of the results, the authors propose to unify various existing models with similar characteristics, obtaining as a result high correlation coefficients that support their validity. They also recommend developing future models applied to Colombian conditions with one lane or bidirectional roadways as well as four-lane ones.
\end{abstract}

Keywords: consistency, geometric design, operative speed, speed of design, speed profile.

\section{CONSISTÊNCIA NO DESENHO: PREDIÇÃO DA VELOCIDADE DE OPERAÇÃO EM RODOVIAS}

Resumo. A consistência é um conceito utilizado, entre outros, para avaliar parte da segurança nas rodovias, tanto na fase de desenho quanto na de operação; por exemplo, identificando a variação da velocidade de operação a respeito da velocidade de desenho ao longo de uma rodovia. Neste artigo, apresenta-se o conceito de consistência no desenho de rodovias e seus diferentes enfoques e mostram-se os principais modelos que têm sido aplicados segundo o perfil de velocidade, o qual se baseia na velocidade de operação em que se observa que os motoristas operam seus veículos em condições de fluxo livre ao longo da rodovia. Com o objetivo de simplificar os modelos de predição de velocidade de operação, mas sem perder a qualidade dos resultados, apresenta-se uma proposta para unificar vários dos modelos existentes que possuam características similares, obtendo como resultado altos coeficientes de correlação que suportam sua validade. Além disso, recomenda-se desenvolver futuros modelos aplicados às condições colombianas para rodovias com via simples bidirecional ou unidirecional e para vias duplas.

Palavras-chave: consistência, desenho geométrico, velocidade de operação, velocidade de desenho, perfil de velocidade. 


\section{Introducción}

Todo proyecto de ingeniería debe cumplir con tres aspectos fundamentales: seguridad, comodidad y economía. Indudablemente, los tres deben ser tenidos en cuenta en los proyectos viales, aunque la seguridad es el más importante: esta debe ser evaluada de diferentes maneras durante el proceso de diseño, y es la consistencia una de las técnicas más utilizadas para ello.

La consistencia en el diseño geométrico se refiere a que los cambios de velocidad de operación que se experimentan a lo largo de la carretera no sean fuertes y que dicha velocidad no difiera fuertemente de la velocidad de diseño, que se debe elegir según la función de la carretera y las expectativas del conductor. La evaluación de la consistencia en el diseño geométrico de carreteras se realiza con el fin de identificar aspectos relacionados con la seguridad de la circulación de los vehículos, con lo que se pueden plantear cambios para mejorar la circulación vehicular.

Las principales áreas de análisis para la consistencia en el diseño geométrico son consideraciones de velocidad, seguridad y desempeño. El primero se refiere a predicciones de la velocidad de operación; el segundo estudia los accidentes de tránsito, la estabilidad de los vehículos y las mejoras de bajo costo; las consideraciones de desempeño contemplan, entre otros, la carga de trabajo del conductor, la predisposición del conductor y diseños de la vía [1].

En las consideraciones de la velocidad, las técnicas utilizadas son: modelos de perfil de velocidad, índices de alineamiento y medidas de distribución de la velocidad. La más utilizada es la primera, en la que se valoran, entre otros, factores de geometría, características del conductor y del vehículo.

En este trabajo se hace énfasis en los modelos de perfil de velocidad que usan la velocidad de operación (concepto más utilizado), que corresponde a la velocidad a la cual se observa que los conductores operan sus vehículos en condiciones de flujo libre, y que equivale al percentil 85 de la distribución de velocidades observadas en un sitio en particular [2].

\section{Estado del arte - Marco teórico}

Las medidas de la consistencia de diseño se clasifican en dos categorías: consistencia del sistema, que se refiere a la medida de la consistencia entre los elementos, y consistencia local que se mide dentro de los elementos [3].

Es posible definir la consistencia en el diseño como los cambios poco notorios entre la velocidad de diseño y la velocidad de operación experimentada por el conductor, la cual es elegida de acuerdo con las expectativas de este [4], [5]. Un diseño inconsistente se define como una característica, o una combinación de características geométricas inusuales o extremas que pueden obligar a los conductores a operar de una manera insegura [4], [6].

Existen tres tipos de enfoques para desarrollar los modelos de evaluación de la consistencia en el diseño: según la velocidad, la seguridad y el desempeño, como se observa en la figura 1 [1]. Los modelos según la velocidad están basados en la predicción de la velocidad de operación, cuya predicción está afectada por factores como radios de curvatura, longitud de la curva horizontal, distancia de visibilidad, peralte, factor de fricción lateral y otras condiciones del pavimento.

Los modelos según la seguridad asocian la consistencia con la operación segura del tráfico, con el desempeño eficiente del conductor y con el diseño geométrico de la vía. La seguridad de las carreteras generalmente se mide de acuerdo con la cantidad de accidentes de tráfico que ocurren por diferentes factores geométricos, como son: intersecciones en cruz, radio de las curvas horizontales, pendiente, distancia de visibilidad y volumen vehicular. Los modelos según el desempeño se basan en las consecuencias que tiene el diseño inconsistente en la actitud del conductor volviendo insegura e ineficiente la manera de conducir; los factores que influyen en este enfoque son la cantidad de acciones de conducción que se tienen que desarrollar en cierta cantidad de tiempo, distancia de invisibilidad que tiene el conductor para anticiparse a lo que debe hacer y elementos estéticamente desagradables en la vía. 


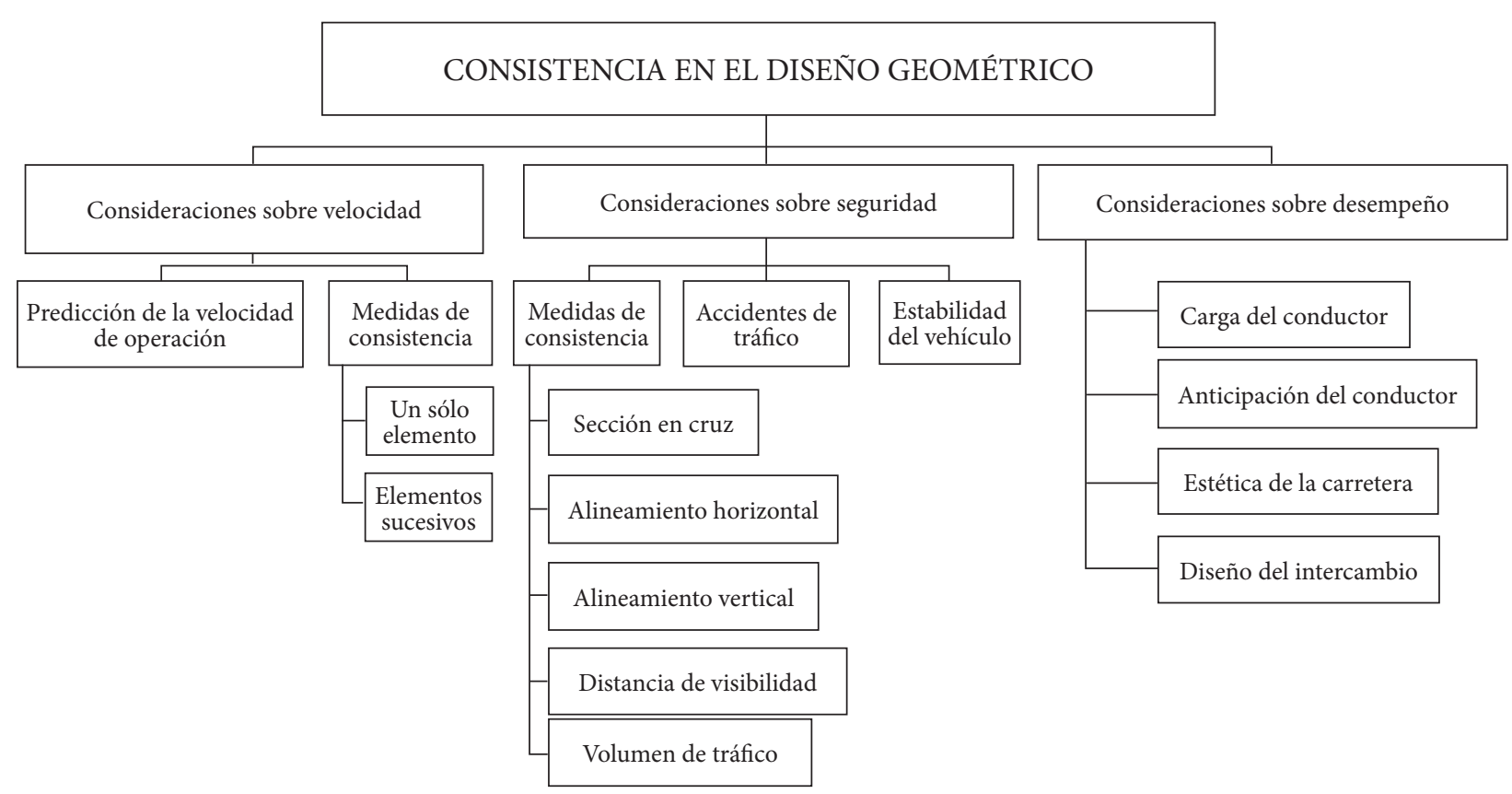

Figura 1. Principales áreas de la consistencia en el diseño geométrico Fuente: [1] y elaboración propia

\subsection{Consistencia en el diseño geométrico}

Se han desarrollado varios métodos y modelos, y hay marcado consenso en que la principal herramienta para evaluar la consistencia puede ser la velocidad de diseño, pues con ella se restringen inconsistencias geométricas. Pero en la actualidad el método más utilizado es el de modelos de perfil de velocidad basados en la velocidad de operación, en las que para un solo elemento geométrico la consistencia se evalúa comparando la velocidad de diseño $(\mathrm{Vd})$ y la velocidad de operación $\left(\mathrm{V}_{85}\right)$; mientras que para elementos geométricos sucesivos la consistencia se evalúa con la velocidad de operación de estos elementos; es precisamente sobre este método que trata este artículo.

(a)

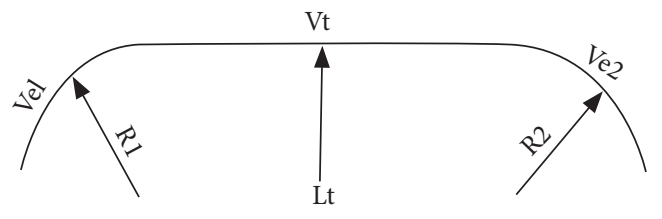

Un perfil de velocidad es básicamente un gráfico de velocidades de operación en el eje vertical frente a la distancia a lo largo de la calzada en el eje horizontal. Este perfil se obtiene midiendo las velocidades de operación de los elementos geométricos sucesivos, de los alineamientos horizontal y vertical. Para el caso de carreteras no existentes o en proyecto, este perfil de velocidad se obtiene mediante métodos de regresión que relacionan la velocidad de operación y las características geométricas [7]; en el caso de las carreteras existentes, se obtienen mediante la distribución de velocidades de los usuarios. En la figura 2, se muestra un ejemplo de lo que es un perfil de velocidad.

En los modelos de perfil de velocidad se utiliza el concepto de velocidad de operación para analizar la

(b)

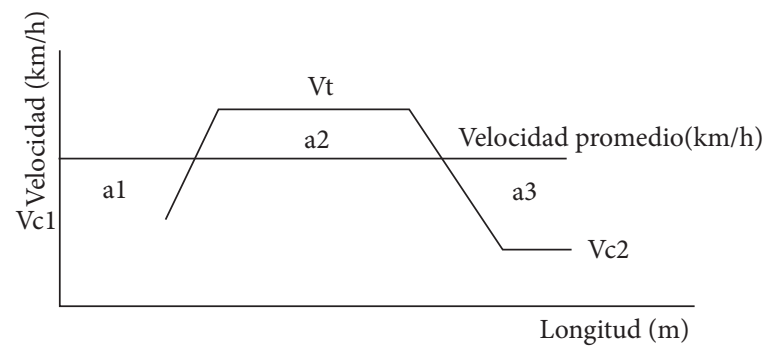

Figura 2. Ejemplo de una sección de vía (a) y su perfil de velocidad (b) Fuente: [8] y elaboración propia 
consistencia en el diseño, pues es la velocidad que muestra el desempeño real de los conductores, a la cual los conductores operan sus vehículos en condiciones de flujo libre. La medida más frecuentemente utilizada de la velocidad de operación, que corresponde al percentil 85 de la distribución de velocidades observadas asociadas a un sitio de la carretera y sus condiciones geométricas [2]. Cuando en el perfil de velocidad hay grandes diferencias de velocidades de operación entre las características sucesivas del alineamiento, se dice que hay inconsistencias en el diseño [9].

Se han desarrollado varias metodologías para predecir la velocidad de operación. Entre estos se cita el método suizo como el más antiguo, aplicado solamente a vías rurales, que busca identificar las diferencias de velocidad entre elementos geométricos sucesivos mediante un perfil de velocidades que tiene en cuenta tres aspectos para considerar consistente un alineamiento: la máxima diferencia de velocidad entre una curva y una tangente es $5 \mathrm{~km} / \mathrm{h}$, la máxima diferencia de velocidad entre dos curvas sucesivas es $10 \mathrm{~km} / \mathrm{h}$ y la distancia de visibilidad no debe ser menor que la requerida para que el cambio de velocidad entre curvas sucesivas sea de $0,8 \mathrm{~km} / \mathrm{h}[10]$.

Otro modelo es el alemán, que utiliza la tasa de cambio de curvatura (Curvature Change Rate - CCR) para medir la homogeneidad de la carretera, definida como "el cambio angular absoluto en dirección horizontal por unidad de distancia" [10].

Lamm et al. desarrollaron un modelo en el cual sugieren usar la tasa de cambio de curvatura (CCR) para predecir la velocidad de operación y para localizar inconsistencias de velocidad en alineamientos horizontales [1]. Su modelo llevó a la clasificación del diseño de carreteras en tres categorías: buen diseño (consistente) en el que $\mathrm{CCR}<5$; diseño razonable en el que $5<\mathrm{CCR}$ $<10$; y diseño pobre (inconsistente) para CCR $>10$ [9].

Krammes et al. plantearon un modelo (modificación del suizo) a través del cual descubrieron que cuando la velocidad de diseño es menor o igual a $90 \mathrm{~km} / \mathrm{h}$ es excedida por la velocidad de operación en curvas horizontales [9].

Fitzpatrick et al. [9] se basaron en el modelo de Lamm et al. [1] para evaluar la consistencia en el diseño y desarrollar ecuaciones de predicción de la velocidad combinando alineamiento horizontal y vertical, para validar el perfil de velocidades; posteriormente se incluyó en el programa computacional IHSDM (Interactive Highway Safety Design Model) para evaluar seguridad en carreteras.

En el IHSDM se tiene el Módulo de Consistencia en el Diseño, que calcula el percentil 85 esperado de la velocidad por medio de un modelo de perfil de velocidad, y así estima su diferencia con la velocidad de diseño a lo largo de la carretera y la reducción de la velocidad entre elementos consecutivos [11]. El procedimiento para definir el perfil de velocidad consiste en elegir la velocidad libre en tramos rectos, luego estimar la $V$ en curvas horizontales para ajustarla por aceleración y desaceleración, re-calcular la velocidad de acuerdo con las pendientes de la carretera, determinar el perfil de velocidad definitivo, y por último hacer el ajuste de la velocida al inicio y final del tramo considerado [12].

\section{Modelos para perfil de velocidad}

Varias metodologías para predecir la velocidad de operación consideran la tasa de cambio de curvatura [10]. Lamm et al. definen la tasa de cambio de curvatura como la suma absoluta de los cambios angulares por unidad de longitud así [1]:

$$
C C R=\frac{57300}{L_{t}}\left(\sum_{i} \frac{L c_{i}}{r_{i}}+\sum_{j} \frac{L_{j}}{2 r_{i}}\right)
$$

Donde CCR es tasa de cambio de curvatura (grados/km); $L c i$, longitud de la curva circular $i(\mathrm{~m}) ; L j$, longitud de la curva espiral $j(\mathrm{~m}) ; r$, radio de la curva circular $i(\mathrm{~m})$; y $L t$, longitud total de la sección $(\mathrm{m})$.

En la tabla 1 se presentan los modelos para la predicción de la velocidad de operación de vehículos, según diferentes autores; la mayoría de estos proponen el uso de características geométricas de la vía como variables independientes para el cálculo de la velocidad, siendo las más utilizadas el radio de la curva circular (r) en metros y el grado de curvatura (DC) en $\% 100 \mathrm{~m}$, otros autores proponen variables como longitud de la curva circular $(L c)$ en metros, el ángulo total girado $(\mathrm{O})$ en ${ }^{\circ}$, tasa de cambio de curvatura (CCR) en $\% \mathrm{~m}$ y velocidad de la tangente anterior (VAT) en $\mathrm{km} / \mathrm{h}$. Todos los modelos fueron desarrollados en carreteras interurbanas de 2 carriles, sin paso por zona urbana o intersecciones [13]. 
Tabla 1. Modelos para la predicción de la velocidad de operación

\begin{tabular}{|c|c|c|c|c|c|}
\hline Modelo & $\begin{array}{l}\text { Condiciones geométricas } \\
\text { alineamiento horizontal y } \\
\text { vertical }\end{array}$ & Autores & Año & País & Ref \\
\hline$V_{85}=35,086-289,99 / \sqrt{r}+0,759 V A T$ & & Cardozo et al. & 1998 & Varios & {$[13]$} \\
\hline$V_{85}=120,16-5596,72 / r$ & & Castro et al. & 2008 & España & {$[13]$} \\
\hline$V_{85}=102,70-(730,39 / r)-(1498,90 / L c)$ & $\begin{array}{l}\text { Curvas horizontales con curvas } \\
\text { verticales cóncavas, radio }<300 \\
\mathrm{~m}\end{array}$ & Castro et al. & & Colombia & [13] \\
\hline$V_{85}=35,43+0,219 r$ & $\begin{array}{l}\text { Curvas horizontales con radio }< \\
300 \mathrm{~m} ;-9 \%=\text { pendiente }=-4 \%\end{array}$ & Castro et al. & & Colombia & {$[13]$} \\
\hline$V_{85}=105,08-149,69 / K$ & \begin{tabular}{|l|} 
Curvas verticales convexas con \\
visibilidad limitada $(\mathrm{K}=43 \mathrm{~m} / \%)$ \\
en tangente horizontal
\end{tabular} & Castro et al. & 2000 & USA & {$[13]$} \\
\hline$V_{85}=104,82-3574,51 / r$ & $\begin{array}{l}\text { Curva horizontal; } 0 \%= \\
\text { Pendiente }<4 \%\end{array}$ & Fitzpatrick et al. & 2000 & USA & {$[13]$} \\
\hline$V_{85}=105,98-3709,90 / r$ & $\begin{array}{l}\text { Curva horizontal; }-4 \%= \\
\text { Pendiente }<0 \%\end{array}$ & Fitzpatrick et al. & 2000 & USA & {$[13]$} \\
\hline$V_{85}=102,10-3077,13 / r$ & $\begin{array}{l}\text { Curva horizontal; }-9 \%= \\
\text { Pendiente }<-4 \%\end{array}$ & Fitzpatrick et al. & 2000 & USA & [13] \\
\hline$V_{85}=103,24-3576,51 / r$ & $\begin{array}{l}\text { Curva horizontal con visibilidad } \\
\text { limitada por curva vertical }(\mathrm{K}= \\
43 \mathrm{~m} / \%)\end{array}$ & Fitzpatrick et al. & 2000 & USA & {$[13]$} \\
\hline$V_{85}=105,32-3438,19 / r$ & $\begin{array}{l}\text { Curva horizontal combinada con } \\
\text { curva vertical cóncava }\end{array}$ & Fitzpatrick et al. & 2000 & USA & {$[13]$} \\
\hline$V_{85}=96,61-2752,19 / r$ & $\begin{array}{l}\text { Curva horizontal; } 4 \%= \\
\text { Pendiente }<9 \%\end{array}$ & Fitzpatrick et al. & 2000 & USA & {$[13]$} \\
\hline $\mathrm{V}_{85}=$ Velocidad deseada asumida & $\begin{array}{l}\text { Curva vertical cóncava en } \\
\text { tangente horizontal }\end{array}$ & Fitzpatrick et al. & 2000 & USA & {$[13]$} \\
\hline $\mathrm{V}_{85}=$ Velocidad deseada asumida & $\begin{array}{l}(\mathrm{K}>43 \mathrm{~m} / \%) \text { en tangente } \\
\text { horizontal }\end{array}$ & Fitzpatrick et al. & 2000 & USA & {$[13]$} \\
\hline $\mathrm{V}_{85}=$ Velocidad deseada asumida & $\begin{array}{l}\text { Curva vertical convexa sin límite } \\
\text { de distancia de visibilidad }\end{array}$ & Fitzpatrick et al. & 2000 & USA & [13] \\
\hline $\mathrm{V}_{85}=$ Velocidad deseada asumida & $\begin{array}{l}\text { Curva horizontal con curva } \\
\text { vertical convexa sin límite de } \\
\text { visibilidad }\end{array}$ & Fitzpatrick et al. & 2000 & USA & {$[13]$} \\
\hline $\begin{array}{l}\mathrm{V}_{85}=\text { Menor valor obtenido para curvas con pendientes } \\
\text { entre }-9 \% \text { y } 0 \% \text { (descenso) o entre } 0 \text { y } 9 \% \text { (ascenso) }\end{array}$ & & Fitzpatrick et al. & 2001 & USA & {$[13]$} \\
\hline$V_{85}=102,2-0,10 \Omega$ & & Gibreel et al. & 1994 & USA & {$[13]$} \\
\hline$V_{85} B P=95,41-0,45 D C-0,001 D C^{2}$ & & Islam y Seneviratne & 1994 & USA & {$[13]$} \\
\hline$V_{85} E P=103,03-0,73 D C-0,003 D C^{2}$ & & Islam y Seneviratne & 1994 & USA & {$[13]$} \\
\hline$V_{85} M P=96,11-0,32 D C$ & & Islam y Seneviratne & 1990 & USA & {$[13]$} \\
\hline$V_{85}=129,88-623,1 / \sqrt{r}$ & & Kanellaidis et al. & 1995 & USA & {$[13]$} \\
\hline$V_{85}=102,44-2471,81 / r+0,012 L c-0,1 \Omega$ & & Krammes et al. & 1987 & USA & {$[13]$} \\
\hline$V_{85}=95,78-0,076 C C R$ & & Lamm et al. & 1987 & USA & {$[13]$} \\
\hline$V_{85}=96,152-0,302 D C$ & & Lamm et al. & 1990 & USA & {$[13]$} \\
\hline
\end{tabular}




\begin{tabular}{|c|c|c|c|c|c|}
\hline Modelo & $\begin{array}{l}\text { Condiciones geométricas } \\
\text { alineamiento horizontal y } \\
\text { vertical }\end{array}$ & Autores & Año & País & Ref \\
\hline$V_{85}=94,398-3188,656 / r$ & $\begin{array}{l}\text { Curva horizontal; } 0 \%= \\
\text { Pendiente }<4 \%\end{array}$ & Lamm et al. & 1997 & USA & {$[13]$} \\
\hline$V_{85}=103,66-1,95 D C$ & & $\begin{array}{l}\text { McFadden y } \\
\text { Elefteriadou }\end{array}$ & 1997 & usa & [13] \\
\hline $\begin{array}{c}V_{85}=41,62-1,29 D C+0,0049 L c-0,12 \Omega \\
+0,95 V A T\end{array}$ & & $\begin{array}{l}\text { McFadden y } \\
\text { Elefteriadou }\end{array}$ & 2005 & USA & [14] \\
\hline$V_{85}=91,85+9,81 \times 10^{-3} r$ & & Misaghi y Hassan & 2005 & USA & {$[14]$} \\
\hline$V_{85}=94,30+8,67 \times 10^{-6} r^{2}$ & & Misaghi y Hassan & 1994 & Canadá & {$[13]$} \\
\hline$V_{85}=\exp (4,561-0,0058 D C)$ & & Morrall y Talarico & 2000 & usa & {$[13]$} \\
\hline$V_{85}=103,66-1,95 D C$ & & Ottesen y Krames & 2000 & USA & [13] \\
\hline $\begin{array}{c}V_{85}=102,44-1,57 D C+0,012 L c \\
-0,01 D C L c\end{array}$ & & Ottesen y Krames & 2000 & USA & [13] \\
\hline $\begin{aligned} V_{85}=41,62 & -1,29 D C+0,0049 L c \\
& -0,12 D C L c+0,95 V A T\end{aligned}$ & & Ottesen y Krames & 2004 & USA & [13] \\
\hline$C=2,808 \times \exp ^{-0,278 \times\left[\operatorname{Rax} \times\left(\frac{D e s v}{3}, 6\right)\right]}$ & & $\begin{array}{l}\text { Polus y Mattar- } \\
\text { Habib }\end{array}$ & 1996 & Israel & {$[1]$} \\
\hline $\begin{array}{c}V_{85}=99,61-2951, \frac{37}{r}+0,014 L c-0,13 \Omega \\
-71,82 e\end{array}$ & & Voigt & & USA & [13] \\
\hline
\end{tabular}

Fuente: elaboración propia

Los modelos de Lamm et al. y Fitzpatrick et al. para la velocidad de operación de los vehículos en curvas horizontales con pendientes entre $0 \%$ y $4 \%$ funcionan adecuadamente para radios mayores a $35 \mathrm{~m}$, sin embargo, para radios mayores a $300 \mathrm{~m}$ el modelo no presenta variaciones apreciables [13]. En la figura 3 se muestra el comportamiento de estos modelos, para radios entre 35 y $100 \mathrm{~m}$.

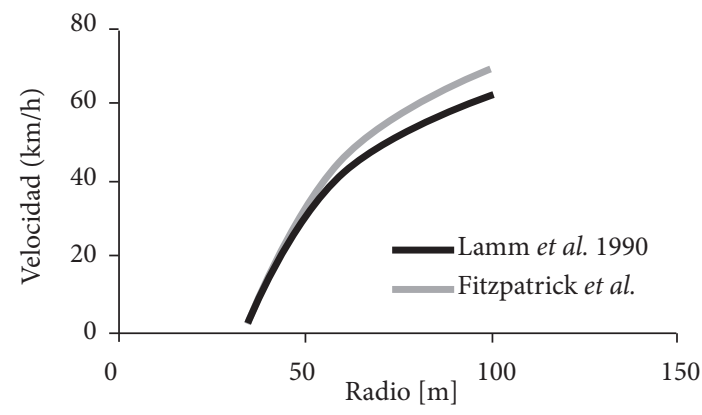

Figura 3. Modelo de Lamm et al. y Fitzpatrick et al. para la velocidad de operación Fuente: elaboración propia
En la figura 4 se muestran los modelos de velocidad de operación de [9] y [13], que aplican para curvas horizontales con pendientes entre $-9 \%$ y $-4 \%$, considerando radios de curva entre 45 y $300 \mathrm{~m}$. El comportamiento de estos modelos es similar a los presentados previamente en la figura 3 haciendo énfasis en que funcionan adecuadamente para radios mayores a $35 \mathrm{~m}$ y que para radios mayores a $300 \mathrm{~m}$ se vuelven asintóticos, es decir que la velocidad es prácticamente constante.

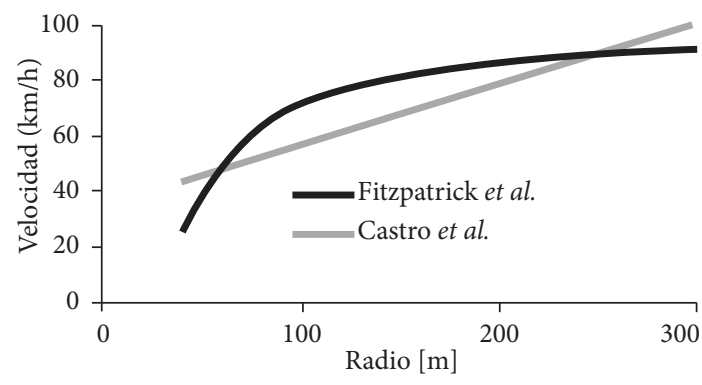

Figura 4. Modelos de velocidad de operación de Fitzpatrick et al. y Castro et al.

Fuente: elaboración propia 


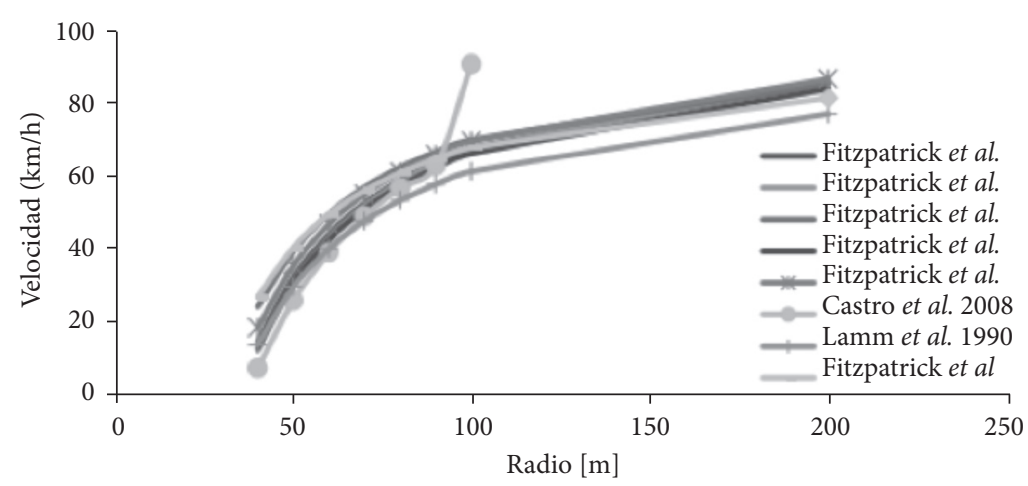

Figura 5. Modelos de velocidad de operación de Fitzpatrick et al., Castro et al. y Lamm et al. Fuente: elaboración propia

En la figura 5 se presentan ocho modelos, seis de ellos fueron propuestos por Fitzpatrick et al. y los otros por Castro et al. y Lamm et al., para diferentes condiciones geométricas de la carretera [12]. Estos modelos se comportan adecuadamente para radios mayores a 40 $\mathrm{m}$; sin embargo, para radios superiores a $200 \mathrm{~m}$ los modelos no varían significativamente. El modelo propuesto por Castro et al. presenta comportamiento adecuado para radios entre 40 y $100 \mathrm{~m}$ [13].
En la figura 6 se presentan los resultados obtenidos para algunos modelos que predicen la velocidad de operación según el grado de curvatura, definido como el ángulo central subtendido por un arco de $100 \mathrm{~m}$. Estos modelos proporcionan resultados lógicos para grados de curvatura en rango definido para cada uno de ellos, se observan diferencias apreciables en resultados para situaciones similares.

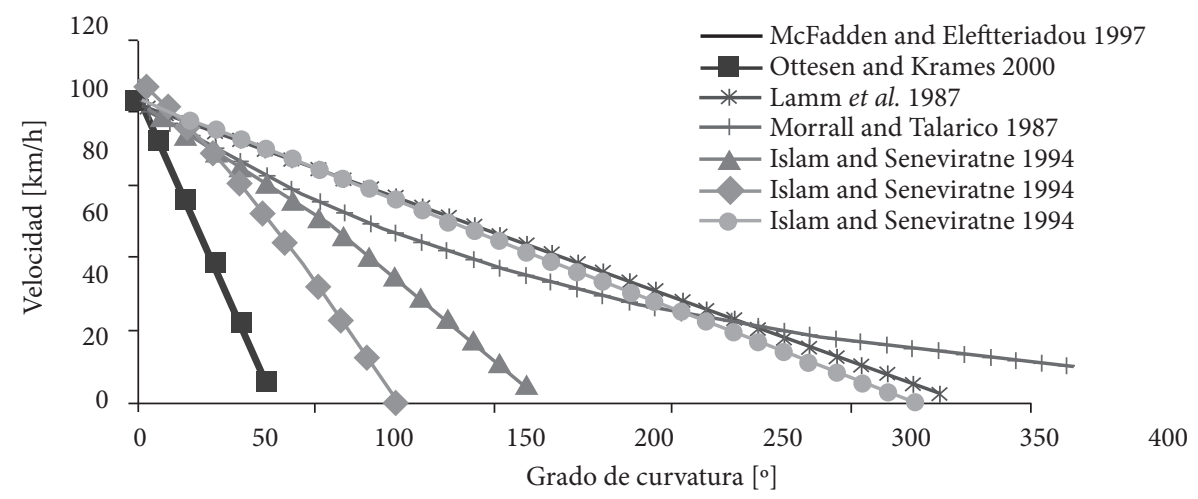

Figura 6. Modelos de velocidad de operación según grado de curvatura

Fuente: elaboración propia

\subsection{Propuesta de modelos}

Como se mostró anteriormente, existen numerosos modelos para predecir la velocidad de operación de los vehículos en las carreteras. Muchos de estos poseen características similares para su aplicación, por lo que a continuación se presenta una propuesta para agrupar o unificar varios de ellos con la finalidad de lograr mayor simplicidad, sin perder la calidad de los resultados obtenidos con ellos en forma independiente.
En la figura 7 se presentan los modelos de Lamm et al. y Fitzpatrick et al. para la velocidad de los vehículos en curvas horizontales con pendientes entre $0 \%$ y $4 \%$ [12] (véase también figura 3). Mediante regresión de mínimos cuadrados, se obtiene el siguiente modelo que unifica los mencionados.

$$
V_{85}=99,609-3381,6 x \frac{1}{r}
$$




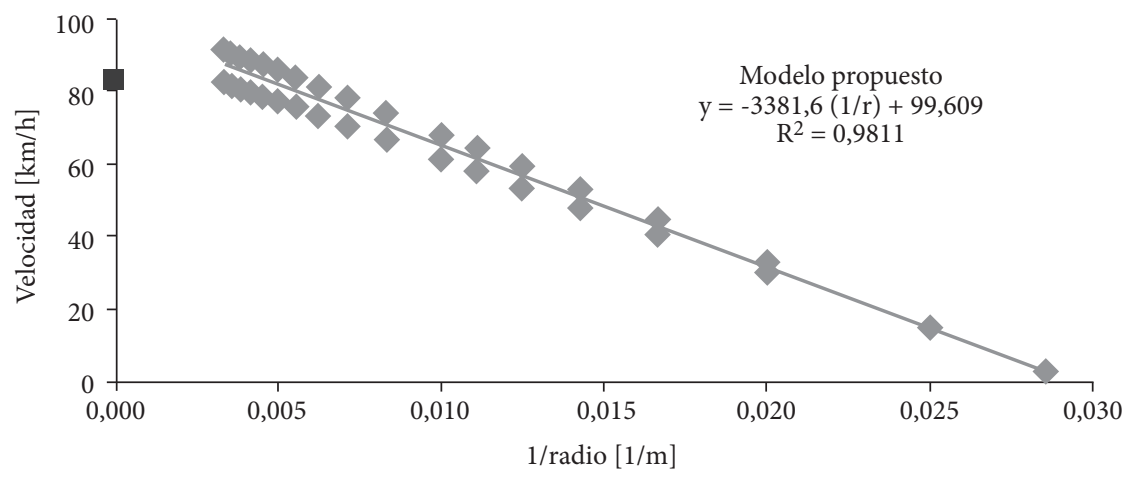

Figura 7. Modelos de Lamm et al. y Fitzpatrick et al. para velocidad de operación en curva horizontal y pendientes entre 0\% y 4\% [12], y modelo propuesto de unificación

Fuente: elaboración propia

Analizando el modelo ajustado, se observa que no presenta variaciones superiores al $6 \%$, es decir, mayores de $5 \mathrm{~km} / \mathrm{h}$ con los modelos de Lamm et al. y Fitzpatrick et al. [13].

En la figura 8 se muestra una modelación lineal de los modelos presentados en la figura 5. Se obtuvo una línea de tendencia de tipo lineal y a su vez un modelo unificado con un coeficiente de correlación igual a 0,98. Se exceptuó el modelo de Castro et al. porque no tiene buen ajuste [13].

$$
V_{85}=101,97-3342,1 \times \frac{1}{r}
$$

Sin embargo, se presentan variaciones hasta de 10 $\mathrm{km} / \mathrm{h}$ en relación con los modelos considerados.

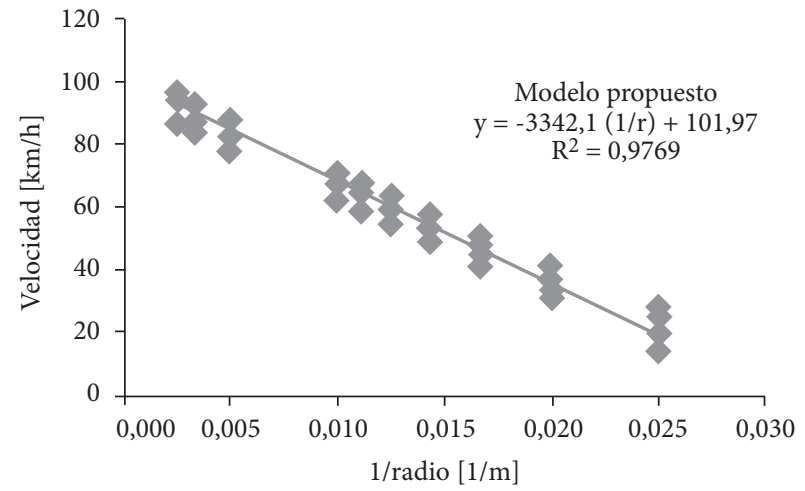

Figura 8. Modelos de velocidad de operación de Fitzpatrick et al. y Lamm et al., y propuesta de unificación Fuente: elaboración propia
En la figura 9 se muestran los resultados gráficos de los modelos de velocidad de operación en curvas horizontales con pendientes entre $0 \%$ y $4 \%$, y pendientes de $-9 \%$ a $-4 \%$. Con la técnica de mínimos cuadrados, se obtiene un modelo que permite agrupar en forma adecuada los que se han considerado, con la siguiente expresión:

$$
V_{85}=100,44-3280,1 \times \frac{1}{r}
$$

Aunque se tiene buen coeficiente de correlación de 0,97 , los resultados obtenidos presentan variaciones porcentuales hasta del $27 \%$, es decir, mayores a $6 \mathrm{~km} / \mathrm{h}$.

\section{Conclusiones}

El estudio y análisis de los modelos permite identificar que en algunos se obtienen valores negativos de la velocidad lo que indica que cada modelo expone un rango de variables en el cual funciona adecuadamente. La mayoría de los modelos analizados presentan un comportamiento adecuado para curvas con radios mayores a $40 \mathrm{~m}$ y menores a $300 \mathrm{~m}$ aproximadamente.

Se pudieron encontrar modelos para predecir la velocidad de operación de los vehículos, que unifican algunos existentes, con altos coeficientes de correlación indicando su validez; sin embargo, algunos presentan variaciones superiores a $10 \mathrm{~km} / \mathrm{h}$ con respecto al modelo original. Se determina que el modelo unificado que mejor se ajusta a los modelos originales es el que relaciona curvas horizontales con pendientes entre $0 \%$ y $4 \%$, que muestra variación no mayor a $5 \mathrm{~km} / \mathrm{h}$. 


\section{Recomendaciones}

Colombia presenta una topografía muy diversa, que varía desde terrenos planos hasta escarpados, requiriendo así parámetros de diseño específicos para cada uno de estos. Por esta razón, no es recomendable utilizar modelos de predicción de velocidad desarrollados en otros países [12] cuyas características topográficas difieren significativamente de las nuestras. Dada la situación, se hace necesario realizar estudios para determinar expresiones matemáticas que modelen la velocidad de operación de los vehículos, para carreteras con calzada sencilla bidireccional o unidireccional, y para dobles calzadas.

\section{Referencias}

[1] G. M. Gibreel, S. M. Easa, Y. Hassan y I. A. El-Dimeery, "State of the art of highway geometric design consistency," J. Transp. Eng., vol. 125, n. ${ }^{\circ} 4$, pp. 305-313, Jul. 1999.

[2] AAshto, A Policy on Geometric Design of Highways and Streets, 6th ed. Washington, DC: AAsHTO, 2011.

[3] S. M. Easa, "Improved speed-profile model for two-lane rural highways," Spec. Issue Innov. Transp. Eng., vol. 30, pp. 1055-1065, Nov. 2003.

[4] K. Fitzpatrick, "Evaluation of Desing Consistency Methods for Two-Lane Rural Highways, Executive Summary," Federal Highway Administration, McLean, VA, Resumen Ejecutivo FHWA-RD-99-173, Aug. 2000.

[5] F. Russo, R. Mauro y G. Dell' Acqua, "Rural highway design consistency evaluation model," Procedia-Soc. Behav. Sci., vol. 53, n. ${ }^{\circ}$ 1877-0428, pp. 953-961, 2012.

[6] S. M. Easa y A. Mehmood, "Establishing highway horizontal alignment to maximize design consistency," NRC Res. Press Web, vol. 34, pp. 1159-1168, Oct. 2007.
[7] A. Jiménez, "Consistencia del trazado de las carreteras," Universidad Nacional de Colombia, sede Medellín, trabajo final de la Especialización en Vías y Transporte, Feb. 2007.

[8] A. Polus y C. Mattar-Habib, "New Consistency Model for Rural Highways and Its Relationship to Safety," Journal of Transportation Engineering, vol. 130, n. ${ }^{\circ}$ 3, pp. 286-293, May 2004.

[9] K. Fitzpatrick et al., "Speed Prediction for Two-Lane Rural Highways," Federal Highway Administration, McLean, VA, FHWA-RD-99-171, Aug. 2000.

[10] L. Cardoso, "Análise crítica de modelos de verificação de consitência de traçado," Tesis de Maestría, Escola Politécnica da Universidade de São Paulo, São Paulo, Br., 2007.

[11] Federal Highway Admininstation (FHwa) and Turner - Fairbank Highway Research Center (TFHRC), Interactive Highway Safety Design Model (IHSDM), Design Consistency Module (DCM) Engineer's Manual, IHSDM Versión 2.05b, DCM Versión 2.01d. McLean, vA: Federal Highway Admininstation (FHWA), 2003.

[12] J. F. Sánchez y M. Castro, "Procedimientos de evaluación de la consistencia del trazado de las carreteras: IHSDM", Rutas: Revista Técnica de Carreteras, n. ${ }^{\circ}$ 96, pp. 5-14, 2003.

[13] M. Castro, J. F. Sánchez, N. Ardila y J. Melo, "Speed Models for Highway Consistency Analysis. A Colombian Case Study", presentado en el 4to Simposio Internacional en Diseño Geométrico de Carreteras, Valencia, España, 2010.

[14] J. F. Sánchez, "Metodología para la evaluación de la consistencia del trazado de carreteras interurbanas de dos carriles", Tesis Doctoral, Universidad Politécnica de Madrid, Madrid, 2011. 